Merkuronitrat berechnet 5 Proz. Stickstoff
0,3841 orgaben
5,10
$0,01960 \mathrm{~g}=$
5,04
$n \quad n$



$0,3782 \mathrm{~g}$ ergaben $0,01295 \mathrm{~g}=3,43$ Proz. Stickstoff

$0,5133 \mathrm{~g}, \quad 0,01750 \mathrm{~g}=3,42$

P yridinnitrat berechneter Salpeter-Stickstoff $"=9,85$ Proz.

$0,4475 \mathrm{~g}$ ergaben $0,04323 \mathrm{~g}=9,72$ Proz. Stickstoff

$0,4673 \mathrm{~g} \# 0,04515 \mathrm{~g}=9,65, \quad$

Diese Analysen beweisen, dafs die Methode für Nitrate brauchbar ist, dagegen scheint sie für Nitrite nicht brauchbar zu sein. So erhielt ich für

'Silbernitrit berechnet 9,09 Proz. Stickstoff

$$
\begin{aligned}
& 0,3092 \mathrm{~g} \text { ergaben } 0,02345 \mathrm{~g}=7,58, ", \\
& 0,5676 \mathrm{~g}, 0,04198 \mathrm{~g}=7,40 ",
\end{aligned}
$$

Dals sich die Methode zum Bestimmen des Stickstoffs im Brunnenwasser eignet, dürfte klar auf der Hand liegen. Zum Titrieren benutze ich $1 / 4$ normal Schwefelsäure und Ammoniak, als vorzliglichen Indikator Fluorescein. Dieses giebt bei einem Tropfen Überschufs obiger Normallösungen den am besten erkennbaren Unterschied. Beim Pyridinnitrat lälst sich dasselbe nicht anwenden, da dieses mit überdestilliert und ein Erkennen des Endpunktes unmöglich macht.

\title{
Coniin und Nikotin
}

von $\mathrm{G}$. $\mathrm{H}$ e u t.

(Eingegangen am 10. April 1893).

Bei der grofsen Aehnlichkeit des Coniins und Nikotins in ihren chemischen Reaktionen dürfte bei forensischen Untersuchungen eine

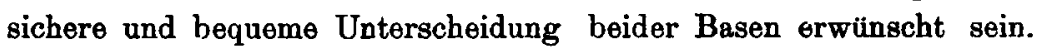
Als unterscheidende Merkmale werden für das Coniin sein Mäuseharn ähnlicher Geruch, die geringere Juöslichkeit in Wasser, dessen geringere Fallbarkeit durch Goldchlorid, Quecksilberchlorid, Platinchlorid, Kaliumquecksilberchlorid, Phosphormolybdänsăure, Gerbsäure und Jodlösung und die Krystallisationsfähigkeit seiner Verbindung mit Chlorwasserstoff angeführt. 
In der Absicht, eine Trennung und Bestimmung des Nikotins bei Gegenwart von Ammoniak zu erzielen, zog ich auch das Coniin in den Kreis moiner. Versuche. Coniin und Nikotin zeigen dem Phenolphtalein gegentiber ein verschiedenes Verhalten. Bringt man zu Nikotin, gelöst in verduinntem Weingeist vom spec. g.0,95- 0,96 , einen Tropfen einer gesättigten alkoholischen Lösung von Phenolphtalein, so tritt keine Rotfärbung der Flüssigkeit ein, während diese auf Zusatz von einer geringen Menge von Coniin sofort zum Vorschein kommt. Noch schärfer zeigt sich dieser Unterschied, wenn man eine wässerige Nikotinlösung mit Phenolphtalein und Chloroform versetzt; bei kräftigem Schutteln verschwindet alsbald die Färbung. Eine wăsserige Coniinlösung verträgt bei gleicher Behandlung den Zusatz selbst des gleichen Volumens Chloroform ohne Beeinflussung der Rotfarbung. - Auf Lackmusfarbstoff dagegen ist Chloroform ohne Einwirkang.

Die quantitative Trennung beider Basen gelingt mit ziemlicher Genauigkeit in folgender Weise:

Man löst die Basen in einer geringen Menge von Wasser unter Zusatz von Alkohol, bis die Flüssigkeit klar erscheint, fugt zu $5 \mathrm{ccm}$ derselben 5 Tropfen Chloroform und einen Tropfen einer gesättigten Phenolphtaleinlösung hinzu und schüttelt das Gemenge tikahtig durch. Alsdann titriert man das Coniin mit $1 / 10$ Normalschwefelsăure bis zur vollständigen Entfarbung der Flüssigkeit unter öfterem Umschütteln, da ein Teil des Coniins im Chloroform sich löst. Schliefslich wird das Nikotin titriert mit Benutzung von Lackmustinktur als Indikator.

Zu nachstehenden Versuchen wurde eine Nikotinlösung benutzt, von welcher $5 \mathrm{ccm} 5,0 \mathrm{ccm} 1 / 10$ Normalschwefelsaure und eine Coniinlösung, von welcher $5 \mathrm{ccm} 16,5 \mathrm{ccm}$ dieser Suure zur Sättigung forderten.

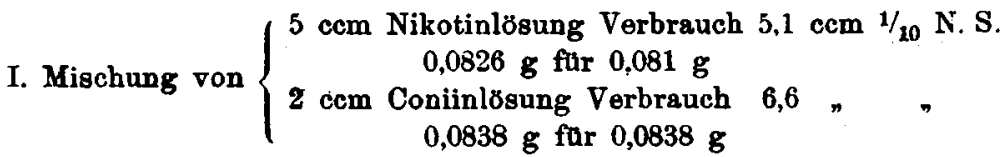

$$
\begin{aligned}
& \text { II. Mischung von }\left\{\begin{array}{c}
5 \mathrm{ccm} \text { Nikotinlösung Verbrauch } 5,1 \mathrm{ccm} 1 / 10 \mathrm{~N} \text {. S. } \\
0,0826 \mathrm{~g} \text { für } 0,081 \mathrm{~g} \\
3 \mathrm{ccm} \text { Coniinlösung Verbrauch } 9,95, \\
0,12636 \mathrm{~g} \text { für } 0,12573 \mathrm{~g}
\end{array}\right.
\end{aligned}
$$


III. Mischung von $\left\{\begin{array}{c}5 \mathrm{ccm} \text { Nikotinlosung Verbrauch } 5,1 \mathrm{ccm} 1 / 10 \mathrm{~N} . \mathrm{S} \text {. } \\ 0,0826 \mathrm{~g} \text { für } 0,081 \mathrm{~g} \\ 10 \mathrm{ccm} \text { Coniinlosung Verbrauch } 32,7 \\ 0,4152 \mathrm{~g} \text { statt } 0,4191 \mathrm{~g}\end{array}\right.$ IV. Mischung von $\left\{\begin{array}{c}10 \mathrm{ccm} \text { Nikotinlösung Verbrauch } 10,0 \mathrm{ccm} 1 / 10 \text { N. S. } \\ 0,262 \mathrm{~g} \text { für } 0,162 \mathrm{~g} \\ 10 \mathrm{ccm} \text { Coniinlosung Verbrauch } 32,8 \\ 0,4165 \mathrm{~g} \text { für } 0,4191 \mathrm{~g}\end{array}\right.$

Arbeitet man mit stark mit Wasser verdünnten Löoungen, so fallen die Bestimmungen sehr ungenau aus, man erhält zu niedrige Werte.

\section{A. Pinner: Ueber Nicotin.}

I. Mitteilung.

(Eingegangen, den 18 April 1893.)

Das lediglich aus Kohlenstoff, Wasserstoft und Stickstoff bestehende Nicotin gehört zu den einfacher zusammengesetzten Alkaloiden und ist, da es leicht in reinem Zustande isoliert werden kann, auch schon frthzeitig rein dargestellt worden. In einer meisterhaft durchgeführten und preisgekrönten Untersuchung haben Posselt und Reimann') 1828 die Base völlig rein bereitet, erst 14 Jahre später haben Ortigosa ${ }^{2}$, kurz daraut Barral ${ }^{3}$ ) dieselbe nebst einigen Salzen analysiert und für sie dis Formel $\mathrm{C}_{10} \mathrm{H}_{16} \mathrm{~N}_{2}$ aufgestellt, während Melsens4) im Jahre 1844 die richtige Zusammen. setzung $\mathrm{C}_{10} \mathrm{H}_{14} \mathrm{~N}_{2}$ ermittelte. Seit dieser Zeit ist das Nicotin häufiger Gegenstand eingehender Untersuchungen gewesen, gleichwohl ist unsere Kenntnis tiber den molekularen Aufbau des Alkaloids bis jetzt ganz aufserordentlich mangelhaft. Schuld an dem verhältnismäfsig geringen Erfolg der zahlreichen mit grolsem Aufwand an Zeit und Geschick durchgeführten Untersuchungen mag einerseits die Schwierig keit gewesen sein, gut krystallisierte und deshalb mit Sicherheit in reinem Zustande herzustellende Derivate zu gewinnen, denn nur

1) Magazin der Pharmacie 24. 138.

2) Annal. der Pharm. 41. 114.

3) Das. 44. 281.

4) Das. 49.353. 